\title{
Visualisation of Multibeam Echosounder Measurement Data
}

\author{
Wojciech Maleika and Piotr Czapiewski \\ West Pomeranian University of Technology, Szczecin, \\ Faculty of Computer Science and Information Technology, \\ Zolnierska 52, 71-210, Szczecin, Poland \\ \{wmaleika,pczapiewski\}@wi.zut.edu.pl
}

\begin{abstract}
During a sea survey session performed using a multibeam echosounder millions of measurement points are generated. Sea surveys should be carried out in such a way, that the maximum accuracy of created seabed models (DTM) is achieved and the standards specified by the IHO S-44 guidelines are met. One of the requirements is so called full sea floor search, which means the ability of a system to detect all the cubic features at least $1 \mathrm{~m}$ in size. Spatial distribution of measurement points is irregular and the distances between closest data points are varying, depending on many factors (on survey parameters, depth or distance between the beam and the vessel). Due to those reasons, it is difficult for the users of hydrographic systems to evaluate the degree of coverage of seabed by measurement points, and therefore to confirm fulfilment of the normative requirements. As a solution we propose visualisation methods for measurement data collected in sea surveys. Specific features of such a visualisation are explained and a method for creating the images is presented, along with some exemplary results.
\end{abstract}

Keywords: data visualization, hydrographic systems, sea surveys.

\section{Introduction}

One of the most important yet also most difficult tasks involved in the process of building spatial information systems is creating a digital terrain model (DTM). This model is composed of the basic information layer used by GIS systems, and is used for either spatial mappings of parameters or assessments. Contemporary users of DTM are very demanding since they require a high quality of data (in terms of accuracy, reliability and validity), efficient data processing and visualization, and the ability to analyse the data in real time [3].

To create a DTM it is necessary to measure depths. Multibeam echosounders record depth observations, obtaining a large amount of data 510] - during one session, the depth and position of millions of points may be recorded. The cardinality and density of the collected data depends on the parameters of the recording device (i.e. the echosounder parameters: number of beams, sampling frequency and beam width; and the survey parameters: speed of the measurement vessel, number of survey tracks, and survey track configuration) 611.

P. Maji et al. (Eds.): PReMI 2013, LNCS 8251, pp. 373-380, 2013.

(C) Springer-Verlag Berlin Heidelberg 2013 
Based on the data collected during sea surveys, DTMs are created using appropriate processing algorithms 89. The created DTMs and derived seabed charts must fulfil the norms specified by the International Hydrographical Organization [7]. The maing goal of this document is to specify the minimal hydrographic survey norms, to ensure the accuracy of the created models and maps. These norms also enable users to determine the spatial uncertainty of the data and effective utilization of them ensures the safe use of measurement results.

One of important parameters described in the regulations is the ability to perform so called full sea floor search. In practice, it is impossible to achieve $100 \%$ bathymetric coverage. Hence, a full sea floor search means a systematic method of exploring the sea floor to detect most of the cubic features of predefined size (1-2m for shallow waters). It should be noted that the IHO Special Order "feature detection requirements" of $1 \mathrm{~m}$ and $2 \mathrm{~m}$ cubes respectively, are minimum requirements. In certain circumstances it may be deemed necessary by the hydrographic offices to detect smaller features to minimise the risk of undetected hazards to surface navigation.

The evaluation of the number and distribution of measurement points, as well as the seabed coverage, is usually performed visually [210]. Contemporary hydrographic and GIS software allow for such a visualisation, but the image is usually created in a simplest way - for each measurement point a corresponding pixel is set in the output image (presented on screen) [11. Since we are dealing with huge amount of data (many millions of points) with high density, usually the imaged area appears solid, without the possibility to distinguish particular points (see Fig. 1a). Only after a significant magnification of a fragment (see Fig. 1b) one can see precisely the number and distribution of points in a given subarea. Using such a solution it is however difficult to assess the distances between points, and hence to determine, if any cubic feature might remain undetected. Also, viewing larger areas in high magnification is cumbersome.

Given the above, it is worthwhile to develop a visualisation method for such data, allowing for a clear indication of places, where the DTM might not conform to IHO standards. The following paper presents two methods for visualising measurement data coming from multibeam echosounder, which aim to simplify the assessment of seabed coverage and to facilitate the detection of places, where the measurements might be insufficient to detect all the required features.
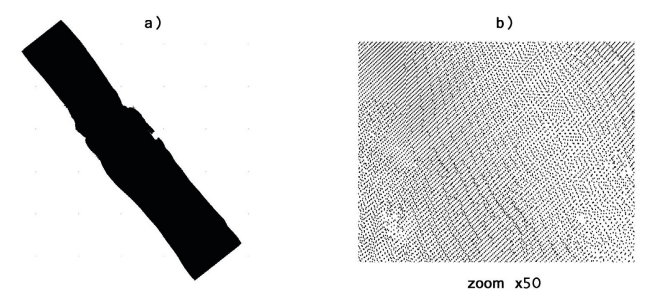

Fig. 1. Visualisation of measurement points in Surfer 9.0 software - whole area (a), a magnified fragment (b) 


\section{A New Method for Visualisation of Measurement Data Distribution}

The measurement points coming from multibeam echosounder are placed in XYZ space ( $\mathrm{X}$ and $\mathrm{Y}$ - position of measurement, $\mathrm{Z}$ - depth). XY values are given in one of reference systems (e.g. WGS84, EUREF-89, system 2000, UTM, etc.) [5]. In case of UTM system, the position of measurement is given in metric values, i.e. we note the distance on the $\mathrm{X}$ and $\mathrm{Y}$ axes from a selected base point. Data files saved in such a format simplify the calculation of distance between points (for other formats appropriate transformations must be performed).

Most hydrographic and GIS software allow for a 3D visualisation of measurement data. Unfortunately, the way the data is presented does not allow to assess the distribution or density of the points. There is also the possibility to present the data on a $2 \mathrm{D}$ image, but the imaging algorithms simply consist in determining for each measurement point a corresponding pixel and setting it to black. With such an approach, often multiple measurement points are mapped into the same image pixel. Furthermore, the size of created image is usually fitted to the software windows size and screen resolution, and does not depend on the size of visualised area. Such a visualisation of data makes it impossible to analyse the measurement data regarding the seabed coverage in a fast and precise way [1.

An important property, which should be considered, is a clear indication of not only the places, where the surveys were performed, but more importantly the places, where the density of measurement data is insufficient, which might result in some features remaining undetected.

Below are listed the basic features, that a visualisation method should possess, in order to allow for the assessment of the correctness of performed survey:

- possibility to define the size of areas or objects, that should always be detected (in metres),

- possibility to define the size of the output image,

- each subarea of a predefined size with no depth measurements performed must be clearly visible in the output image (the point must not be black out as a result of neighbouring points averaging or image downsampling).

The images created in that way will clearly show the places, where the density of data is insufficient and the IHO standard requirements might not be met.

Let us define the following variables:

- grid - the cubic feature's size, which should always be detected,

- $X_{\text {size }}, Y_{\text {size }}$ - the size of output image in pixels,

- $X_{t e m p}, Y_{t e m p}$ - the size of intermediate image (its dimensions are adjusted to the area size and grid size),

- src-file - the name of a file with measurement data,

- $x_{\min }, x_{\max }, y_{\min }, y_{\max }$ - edge values for measurement points (it is assumed, that this data is stored in metric UTM system),

- $x_{\text {data }}, y_{d a t a}$ - the coordinates of subsequent measurement points (UTM),

- $x_{\text {image }}, y_{\text {image }}-$ subsequent pixel coordinates in the image. 
In the presented method, the visualisation process consist of two basic stages. In the first stage, based on measurement data an intermediate high resolution image is created (adjusted to the area and grid size), where one pixel corresponds to a cubic feature size. In the second stage the final image (of size predefined by the user) is created, where the averaged information from the intermediate image is presented, with the addition of indication of areas with insufficient measurement points density.

The size of the intermediate image may be calculated using the formula:

$$
\left\{\begin{array}{l}
X_{\text {temp }}=\frac{X_{\max }-X_{\min }}{g r i d} \\
Y_{\text {temp }}=\frac{Y_{\max }-Y_{\min }}{g r i d}
\end{array} .\right.
$$

Next, for each measurement point the corresponding pixel coordinates in the intermediate image are determined, and the pixel is set:

$$
\left\{\begin{array}{l}
x_{\text {image }}=\frac{\left(x_{\text {data }}-X_{\min }\right)\left(X_{\text {temp }}-1\right)}{X_{\max }-X_{\min }}+1 \\
y_{\text {image }}=\frac{\left(y_{\text {data }}-Y_{\min }\right)\left(Y_{\text {temp }}-1\right)}{Y_{\max }-Y_{\min }}+1
\end{array} .\right.
$$

After this stage the intermediate image contains a detailed map of measurement points distribution. Thanks to adjusting the image size to the size of area under investigation and to the grid size, blank pixels inside the area denote the subareas (the size of the grid) for which no measurements were taken. It could be interpreted as the possibility of missing in those subareas some features of a predefined size, hence the measurements do not comply with IHO regulations.

The dimensions of the intermediate image are usually bigger than the output image. For example, for an area $3 \times 2 \mathrm{~km}$ in size and a grid size of $0.5 \mathrm{~m}$ the dimensions of intermediate image will be $6000 \times 4000$ pixels. The output image, however, should be displayed on screen and fit into software window, hence it will be definitely smaller. The simplest method of creating a smaller output image would be downsampling. Unfortunately, this would cause single white pixels present in the intermediate image to be averaged and not appear on the output image. Such a situation is undesirable, hence the scaling process should be performed in such way, that every white pixel in the intermediate image retains visibility in the output image. This approach will guarantee, that all areas with insufficient measurement data density will be visible in the output image.

The algorithm for creating images depicting spatial distribution of measurement points is presented in the Figure 2.

The results of the developed algorithm are presented using two real datasets from sea surveys, performed with Simrad EM3000 multibeam echosounder [4]. The parameters of test areas named "gate" and "rotator", as well as the dimensions of created intermediate images, are listed in Table 1.

In the Figure 3 a sample visualisation of measurement points distribution created using Surfer 8.0 software is shown (this is a typical visualisation performed using standard algorithms). Figure 4 presents a visualisation created using the method being described here. The images where created for 3 different grid sizes $(1,0.5$ and $0.25 \mathrm{~m})$. The output image has the size of $1300 \mathrm{x} 1700$ pixels. 


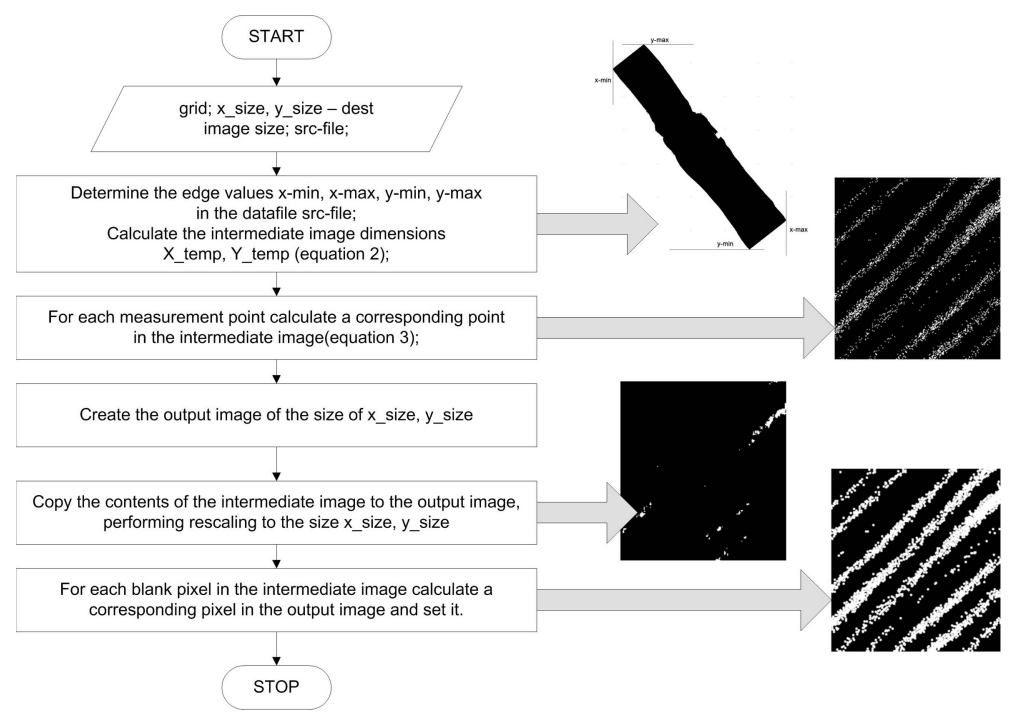

Fig. 2. The algorithm for creating images of measurement points spatial distribution

In case of commonly used today visualisation (see Fig. 3), the pixels denoting measurement points cover $100 \%$ of the area. The user can assume, that a full sea floor search had been achieved, which might not be the case. As we can see on images created using the proposed method, even for grid $=1 \mathrm{~m}$ there are some subareas, where the density of measurement points is too low (seen as dark spots in the image) and where some features located on the sea floor might remain undetected. The IHO regulations state, that most of the cubic features must be detected. It is up to the hydrographer to decide, whether the data density is sufficient or the occurring subareas of low density disqualify the survey.

If we want to make sure, that no objects bigger than $0.5 \mathrm{~m}$ were missed, the visualisation must be performed with grid set to $0.5 \mathrm{~m}$. As we can see in the above images, the number of spots with insufficient density is higher, than for grid $=1$. In such case, the survey probably should not be considered conformant to the regulations. This effect is even more apparent for grid $=0.25 \mathrm{~m}$, where the number of blank spots is extremely high. Additional survey tracks should be performed, in order to gather more data.

A particular property of the images created this way should be noted. One should not assess the incorrect spots quantitatively (e.g. by estimating the percentage of area with insufficient data density). It is caused by the fact, that

Table 1. Properties of measurement data and created intermediate images

\begin{tabular}{|c|c|c|c|c|c|c|}
\hline \multirow{2}{*}{\multicolumn{2}{|c|}{ Name }} & \multirow{2}{*}{$\begin{array}{l}\text { Number of } \\
\text { meas. points }\end{array}$} & \multirow{2}{*}{ Area size $[\mathrm{m}]$} & \multicolumn{3}{|c|}{ Intermediate image size } \\
\hline & & & & $($ grid=1m) & $($ grid $=0.5 \mathrm{~m})$ & $(\operatorname{grid}=0.25 \mathrm{~m})$ \\
\hline 1. & Gate & 3812445 & $811 \times 951$ & $811 \times 951$ & $1622 \times 1902$ & $3244 \times 3804$ \\
\hline 2. & Rotator & 12263261 & $1314 \times 1848$ & $1314 \times 1848$ & $2628 \times 3696$ & $5256 \times 7392$ \\
\hline
\end{tabular}



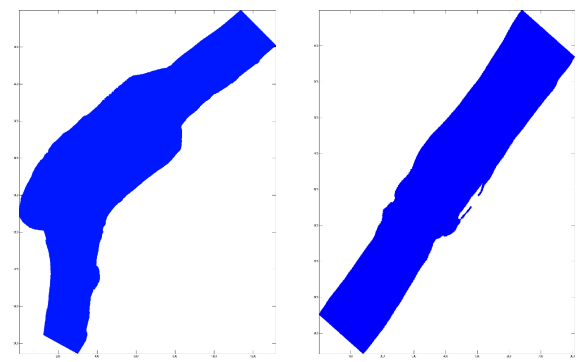

Fig. 3. Measurement points distribution visualisation created in Surfer 8.0 software
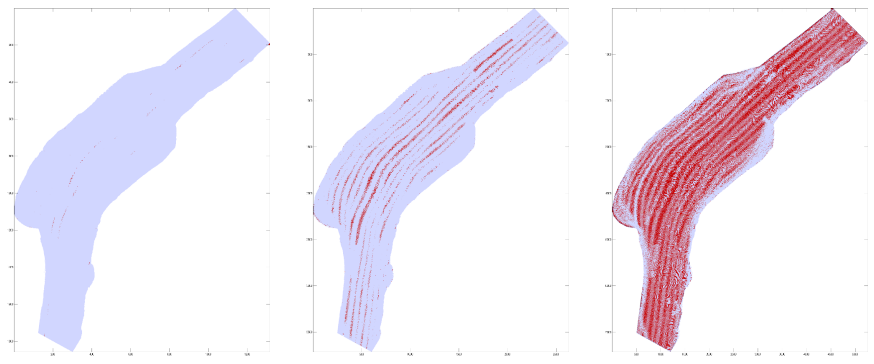

Fig. 4. Visualisation for the "rotator" surface. Grid size: $1 \mathrm{~m}(\mathrm{a}), 0.5 \mathrm{~m}$ (b), $0.25 \mathrm{~m}$ (c)

a single blank pixel in the intermediate image, denoting lack of measurement points in a very small subarea (e.g. $0.0625 \mathrm{~m}^{2}$ for grid $=0.25 \mathrm{~m}$ ), in the output image remains blank, but corresponds to a much bigger area (1-4 $\left.\mathrm{m}^{2}\right)$. In other words, each pixel in the output image corresponds to several pixels in the intermediate image (the number depends on the grid size and dimensions of the output image). If any of those pixels is blank, the pixel in the output image is also blank. This is why those images may be used only to show the locations, where data density is too low.

\section{Quantitative Visualisation of Measurement Points Distribution}

By performing the visualisation using the method described in the previous section, the user obtains only the information on the location of the spots, where the data density is insufficient. Therefore, from the informational point of view, we are dealing with binary images - yes/no, density sufficient/insufficient.

It seems desirable to prepare additional visualisations, which will allow to better assess the distribution of density of measurement points gathered in a given area. This should make it easier for hydrographic and GIS systems' users to perform quantitative analysis of data and to estimate the accuracy of created 

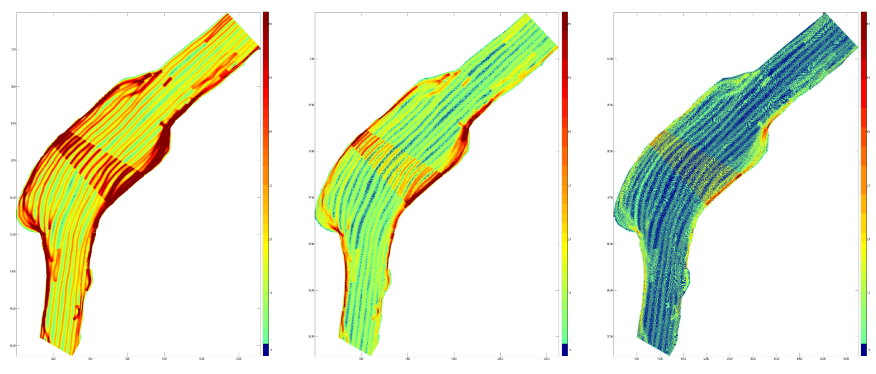

Fig. 5. Quantitative visualisation of the "rotator" surface. Grid size: $1 \mathrm{~m}(\mathrm{a}), 0.5 \mathrm{~m}$ (b), $0.25 \mathrm{~m}(\mathrm{c})$

DTM models. It is obvious, that the bigger the amount of measurement data falling inside a single cell of the model, the lower the mapping error at this point. Quantitative estimation of measurement data allows to assess, whether it is possible to create a precise model, as well as to get additional information on the way the data were gathered.

The method for creating such a type of images is very similar to the one described in section 2. The only modification is making the colour of pixels in the output image dependent on the density of measurement points. In the intermediate image the value of a pixel is equal to the number of the measurement points related to it. In the next step, when determining the value of a pixel in the output image, the mean value of all corresponding pixels from intermediate image is considered. This approach allows for including in the output image the information on the average density of measurement points.

Examples of quantitative visualisation of measurement data are shown in the Figure 5.

In the above examples it is clearly visible, that for a grid size of $1 \mathrm{~m}$, the density of the points near the edges of the areas is equal approximately $50-60$ points $/ \mathrm{m}^{2}$. Inside the areas the density drops to approximately $10-40$ points $/ \mathrm{m}^{2}$. The long red (dark) strips inside the areas show places, where the data from subsequent survey tracks overlap. Thanks to such type of visualisation the user is able to determine the degree of overlapping and the approximate layout of subsequent survey tracks. Also, the places with blank nodes, i.e. subareas with lacking data, are denoted (dark blue/black colour).

For smaller grid sizes (grid $=0.5 \mathrm{~m}$ and grid $=0.25 \mathrm{~m}$ ) a significant decrease in density of measurement points is clearly visible. We can still recognize the distribution of points, layout of survey tracks and occurrences of blank nodes.

The grid size should be adjusted by the user to the size of the area under investigation, the expected accuracy of the model, IHO standards and to the characteristics of the area. For surfaces of low diversity or for typical surfaces, the grid size may be equal $1 \mathrm{~m}$. For particular areas, such as those containing shipwrecks, underwater constructions, port terrains, a more precise grid of 0.5 $\mathrm{m}$ could be used. 


\section{Conclusions}

Users of hydrographic systems do not possess any good tools allowing for visualisation of data coming from multibeam echosounders, so that the interpretation of this data could be easier. The method developed and described here allows for two novel ways of presenting the data, summarised below.

Visualisation of measurement points distribution shows the areas, where the density of measurement points is too low and it is possible to miss some cubic features of a predetermined size. Such type of images allows for quick analysis of measurement data regarding the conformance to IHO regulations.

Quantitative visualisation of measurement data allows for analysis of measurement points density distribution, estimation of survey tracks layout, and hence the assessment, whether the data is sufficient to create a precise DTM.

Institutions that may benefit from the conclusions drawn from the research are those that deal with conducting sea surveys, including bathymetric measurements. The results of the studies could prove useful to those fields in which the accuracy of seabed models is important, such as in oceanographic studies, geology, hydrotechnics, military science and tourism.

Novel imaging techniques might become a part of hydrographic and GIS software, therefore increasing the functionality scope of such software.

\section{References}

1. Bell, J.M., Linnett, L.M.: Simulation and Analysis of Synthetic Sidescan Sonar Images. IEE Proceedings, Radar, Sonar and Navigation (1997)

2. Calder, B.R., Mayer, L.A.: Automatic processing of high-rate, high-density multibeam echosounder data. Geochem. Geophys. Geosyst. 4(6), 1048-1069 (2003)

3. Hamilton, E.L.: Geoacoustic modeling of the sea floor. Journal of the Acoustical Society of America 68(5), 1313-1340 (1980)

4. EM3000 - Multibeam echo sounder. Kongsberg Maritime GmbH (November 2011), http://www.kongsberg-simrad.de

5. Etter, P.: Underwater Acoustic Modeling. Elsevier Applied Science (1991)

6. Gao, J.: Resolution and accuracy of terrain representation by grid DEMs at a microscale. Int. J. Geogr. Inf. Sci. 11(2), 199-212 (2001)

7. International Hydrographic Organization, IHO standards for hydrographic surveys, Publication No. 44, 4th edn. (1998)

8. Lubczonek, J., Stateczny, A.: Concept of neural model of the sea bottom surface. In: Rutkowski, L., Kacprzyk, J. (eds.) Neural Networks and Soft Computing, Advances in Soft Computing, pp. 861-866 (2003)

9. Maleika, W.: Development of a method for the estimation of multibeam echosounder measurement accuracy. Przeglad Elektrotechniczny 88(10b), 205 (2012)

10. Maleika, W., Pałczyński, M., Frejlichowski, D.: Multibeam echosounder simulator applying noise generator for the purpose of sea bottom visualisation. In: Maino, G., Foresti, G.L. (eds.) ICIAP 2011, Part II. LNCS, vol. 6979, pp. 285-293. Springer, Heidelberg (2011)

11. Yanalak, M.: Effect of GRIDding method on digital terrain model profile data based on scattered data. J. Comput. Civil. Eng. 17(1), 58-67 (2003) 